\title{
NSG-70, a new glioblastoma cell line with mixed proneural-mesenchymal features associates NOTCH1-WNT5A signaling with stem cell maintenance and angiogenesis
}

\section{Divya Kumari Singh}

National Centre for Cell Science

Pavan Kumar Mysuru Shivalingappa

National Centre for Cell Science

Aman Sharma

ExoCan Healthcare Technologies Pvt. Ltd.

Abir Mondal

National Centre for Cell Science

Dattatraya Muzumdar

King Edward Memorial Hospital and Seth G.S. Medical College

\section{Anjali Shiras}

National Centre for Cell Science

Sharmila A Bapat ( $\sim$ sabapat@nccs.res.in )

National Centre for Cell Science

\section{Research Article}

Keywords: Glioblastoma, tumor heterogeneity, Glioma subtypes, Glioma stem cells, NOTCH1 signaling, WNT5A signaling

Posted Date: February 21st, 2022

DOI: https://doi.org/10.21203/rs.3.rs-1349869/v1

License: (9) This work is licensed under a Creative Commons Attribution 4.0 International License. Read Full License

Version of Record: A version of this preprint was published at Journal of Neuro-Oncology on April 17th, 2022. See the published version at https://doi.org/10.1007/s11060-022-04002-x. 


\section{Abstract}

\section{Background}

Glioblastoma initiation and progression is believed to be driven by Glioma stem cells (GSCs). Activation of NOTCH1 and WNT, and more recently, non-canonical WNT5A signaling, has been demonstrated to regulate self-renewal and differentiation of the GSCs crucially. High expression levels of NOTCH1 and WNT in GBM tumors contribute to the sustenance of GSCs and mediate characteristic phenotypic plasticity, which is reflected by the different subtypes and tremendous intra-tumor heterogeneity. However, the coregulation of NOTCH1 and WNT5A is not well understood. Here, we studied the role of these molecules in regulating the characteristics of different GSC subtypes.

\section{Methods}

We established a novel GSC-enriched cell model, referred to as NSG-70, from a patient with recurrent GBM. NSG-70 cells harbor a unique cytogenetic feature, viz. isochromosome 9q. At the same time, its expression profiles indicate it to represent a mixed lineage comprising of proneural and mesenchymal subtypes. We examined the relevance of $\mathrm{NOTCH} 1$ and WNT5A signaling and their coordinated action in GBM using these cells and other patient-derived models representing different GSC subtypes.

\section{Results}

Our data revealed that the downregulation of $\mathrm{NOTCH} 1$ resulted in the suppression of stem cell and mesenchymal markers and significantly reduced the levels of WNT5A. NOTCH1 knockdown also led to a notable reduction in the vasculogenic mimicry of GSCs. Interestingly, knockdown of WNT5A exhibited similar effects and drove quiescent GSC towards proliferation. In a complementary manner, ectopic expression of WNT5A or rhWNT5A treatment rescued the effects of NOTCH1 knockdown.

\section{Conclusions}

Given the resistance of GSCs towards conventional therapies in part due to subtype, interconversion demands therapies targeting specific GSC subtype. Our study suggests the need for a combinatorial approach that could effectively target the NOTCH1-WNT5A signaling axis towards eliminating GSCs.

\section{Introduction}

Glioblastoma (GBM) is the most common and malignant tumor of the central nervous system (CNS). It exhibits extensive cellular and molecular heterogeneity which is responsible for treatment failure and tumor recurrence. Several pieces of evidence suggest that GBM is driven by a subpopulation of cancer cells with stem cell characteristics, and which possess a potential for self-renewal and multipotentiality[1, 2]. The survival and self-renewal properties of glioma stem cells (GSCs) is stringently dependent on communication between stem cells and their surrounding cells (stem cell niche) including endothelial and differentiated tumor cells via several tightly regulated signaling pathways. A major 
driving force for regulating self-renewal and proliferative properties of GSCs is mediated by Notch and Wnt signaling [3].

GBM tumors are categorized into four molecular subgroups viz. classical, neural, proneural, and mesenchymal, based on dominant gene expression patterns in each group and are assigned putative prognostic significance[4]. However, most studies identify and characterize two mutually exclusive GSC subtypes: proneural and mesenchymal, with the mesenchymal subtype being more aggressive than proneural. GSCs of all subtypes exhibit phenotypic plasticity, further contributing to the intratumoral heterogeneity[5], besides exhibiting dysregulation of distinct signaling pathways[6], which needs to be studied in detail. Hence, better understanding of GSC subtypes and the signaling pathways maintaining their stemness is indispensable to develop targeted and personalized therapies.

The Notch and canonical Wnt signaling pathways are shown to majorly modulate the motility and invasiveness of hGBM cells by activating an epithelial to mesenchymal-like program in malignant gliomas[7]. They also play an indispensable role in the proliferation of GSCs and neoangiogenesis[8]. Notch signaling pathway is implicated in the maintenance and proliferation of the neural stem cells (NSCs) as well as GSCs[9]. However, how Notch pathway fine tunes its functional mechanisms to bring about the differentiation process or maintenance of stemness in NSCs or GSCs, is largely unknown. Previous reports suggested that several pathways can cross-talk and modulate Notch signaling to suppress its effects on the self-renewal of NSCs/GSCs and induce the differentiation of normal cells or tumor cells[9],[10]. In association with Notch, Wnt/Bcatenin signaling regulates core processes in stem and progenitor cells that include self-renewal, proliferation, differentiation, and migration[11],[12]. Dysregulated Wnt/ßcatenin signaling are frequently observed in several cancers including GBM[12]. However, to our knowledge, there are no reports of cross-talk or coordinated action of NOTCH1 and noncanonical WNT ligand, WNT5A in GSCs. There are evidences indicating overexpression of WNT5A in highgrade gliomas, and a recent study also showed that Wnt5a overexpression induces an infiltrative, mesenchymal-like characteristic in glioma stem-like cells[13]. However, any elaborate study showing its role in regulating the proliferation and migration of GSCs is lacking.

Here, we established and characterized a novel GSC line, referred to as NSG-70, which represents an in vitro model of mixed (proneural and mesenchymal) subtype. Karyotype analysis of NSG-70 GSC displayed several commonly reported chromosomal aberrations along with a unique feature of isochromosome 9q (i9q). Microarray analysis of NSG-70 confirmed the presence of $i 9 q$ and gain of specific cytoband accommodating NOTCH1 gene, besides suggests enhanced expression of additional genes which may confer an advantage to tumor cell growth. This led us to study the biological effect of NOTCH1 signaling in NSG-70 along with two other pre-established GSC lines belonging to different subtypes, from the lab. This led to a comprehensive study of the coregulation of NOTCH1 and WNT5A in modulating the stemness and proliferation potential of GSCs as well as in promoting angiogenesis. Together, these could serve as an unprecedented treatment option for GBM.

\section{Materials And Methods}




\section{Tumor tissue collection and cell culture}

Tumor tissue and normal brain tissue specimens (from epileptic patients) were obtained following approval from Institutional Ethics Committees (IEC) of NCCS, Pune and KEM Hospital, Mumbai, India. Consent of patients was taken before collection of tumor specimens. Tumor grading was performed by neuropathologists according to the World Health organization classification. GSC cultures were established from tumor tissues of histopathologically verified glioblastoma patients. Tissues were mechanically and enzymatically dissociated into single cells and further plated in serum-free medium supplemented with B27, EGF, and bFGF $(20 \mathrm{ng} / \mathrm{ml})$ for enriching stem cells as described earlier[14]. Culture flasks were coated with LDEV-free hESC qualified geltrex (Gibco) for $3 \mathrm{hr}$ at $10 \mathrm{ug} / \mathrm{ml}$ prior to use. Adherent GSC cultures were maintained in serum-free medium as described earlier[15] and dissociated using Stempro Accutase (Gibco). The culture medium was changed twice a week and cultures were observed under the microscope every 24-48 h. Primary cultures were propagated continuously up to 10 to 15 generations without any observed changes in cell morphology and growth characteristics. Human neural stem cell (hNSC) culture was derived from human induced pluripotent stem cells using Gibco pluripotent stem cell (PSC) Neural Induction Medium as per manufacturer's instructions. The medium consists of Neurobasal ${ }^{\circledR}$ Medium and GIBCO ${ }^{\circledR}$ Neural Induction Supplement, 50X (Cat. no. A1647801).

\section{Neurosphere formation assay}

Adherent NSG-70 GSC culture was dissociated and 500 cells were plated in $35 \mathrm{~mm}$ dishes. They were grown in the neurosphere culture conditions as described above for 12-14 days and were fed every 3-4 days by changing half of the medium. Once neurospheres reached approximately $200-300$ cells, they were counted and presented as the percentage neurosphere forming cells of the total 500 cells plated.

\section{Flow cytometry}

Cell surface expression of CD133 and CD44 was examined by flow cytometry using a standard protocol. In brief, the adherent monolayer was dissociated by accutase treatment and harvested as single cell suspension. $2 \mu \mathrm{g} / \mathrm{ml}$ of phycoerythrin (PE)-conjugated anti- CD133, FITC-conjugated CD44 (Miltenyi, Auburn, CA), and a negative control was added to $10^{6}$ cells in $200 \mu \mathrm{l}$ of immunofluorescence buffer (PBS containing $2 \% \mathrm{FBS}$ and $0.02 \%$ sodium azide). After 1 hour of incubation in the dark at $4^{\circ} \mathrm{C}, 10,000$ cells were analyzed using a Becton and Dickinson FACSCanto (Mountain View, CA). The results were processed by using Diva software as the percentage of positive cells.

\section{Confocal microscopy}

Cells were seeded onto coverslips in 24 well plates (BD Bioscience) for $48 \mathrm{~h}$. Confocal imaging was performed using method described earlier [16]. Briefly, cells were fixed with $3.7 \%$ paraformaldehyde (Sigma-Aldrich) followed by permeabilization with $0.01 \%$ Triton X-100, blocking with $5 \%$ BSA, primary antibody (1:100) incubation for $1 \mathrm{~h}$ at room temperature and Alexa Fluor conjugated secondary antibody (1:100) for 1 h. DAPI (4',6-diamidino-2-phenylindole) (Sigma-Aldrich) was used to stain nuclei before 
mounting on slides. The staining was visualized using confocal microscope Leica TCS SP5II (Leica Microsystems CMS, GmbH, Germany).

\section{Tumorigenicity assay}

Animal studies were approved by the Institutional Animal Ethics Committee (IAEC) of NCCS. $1 \times 10^{6}$ of GSCs were injected subcutaneously into NOD-SCID mice. At the end of the experiment, all mice were euthanized and tumor tissues were collected. Finally, tumor volumes were calculated using the formula: $a \times b^{2} / 2$, wherein ' $a$ ' and ' $b$ ' is the longest and the shortest diameter of the subcutaneous tumor that were determined using a vernier caliper.

\section{Pathology and immunohistochemistry}

Hematoxylin and eosin (H\&E) staining and immunohistochemistry were performed on NSG-70 and KW formalin-fixed, paraffin-embedded (FFPE) tissue sections as described[17]. Antigen retrieval was performed by microwave treatment in citrate buffer ( $\mathrm{pH} 6.0)$. Antibodies were used in prescribed dilution, Vectastain Elite ABC kit (Vector Laboratories) and 3,3'-diaminobenzidine (Dako) was used as chromogen for the staining.

\section{RNA extraction and Quantitative real-time PCR}

Total cellular RNA was extracted using TRIzol Reagent (Invitrogen) as per manufacturer's instructions. Quantitative RT-PCR (qRT-PCR) was performed using Power-up SYBR Green (Applied Biosystems, Carlsbad, CA) using QuantStudio 6 Flex Real-Time PCR system (Thermo-Scientific). Primer sequences used are listed in Supplementary Table 1.

\section{Western blotting}

Whole cell lysates were prepared using RIPA protein extraction buffer with 1×protease inhibitor cocktail (Sigma) following manufacturer's instructions. Protein expression was detected using Immobilon Western Chemiluminescent HRP Substrate (Millipore). The details of the primary antibodies used are given in supplementary information.

\section{Cell proliferation assay}

Cell proliferation was determined by MTT assay as described previously. Briefly, $5 \times 10^{3}$ cells of each cell line per well were seeded in a 96 well plate. The absorbance was measured over a period of 5 or 6 days. $20 \mu \mathrm{L}$ of MTT $(5 \mathrm{mg} / \mathrm{mL})$ (Sigma-Aldrich) was added to each well and readings were taken at $0 \mathrm{~h}$ and at every $24 \mathrm{~h}$ interval for six days. The formazan crystals formed were dissolved in dimethyl sulfoxide (DMSO) (Sigma-Aldrich) and absorbance of each well was measured at OD-570 nm in a Bio-Rad microplate reader (Bio-Rad, Hercules, CA, USA).

\section{Cell cycle analysis}


$1 \times 10^{6}$ GSCs were harvested and fixed by adding 70\% ethanol drop-wise while vortexing and stored at $4^{\circ} \mathrm{C}$ for at least 30 minutes. Cells were pelleted at $1200 \mathrm{rpm}$ at room temperature for 5 mins. Cells were rehydrated in PBS for 10 minutes after removing ethanol. GSCs were resuspended in $300 \mu \mathrm{L}$ propidium iodide (PI)/ Triton X-100 staining solution (PBS with $0.1 \%$ Triton X-100, $200 \mu \mathrm{g} / \mathrm{mL}$ DNase-free RNase A, $10 \mu \mathrm{g} / \mathrm{mL} \mathrm{PI}$ ) and incubated for 30 mins in dark. The samples were acquired in FACSCalibur instrument with a $488 \mathrm{~nm}$ excitation laser. The analysis of cell cycle populations was performed in BD CellQuest Pro software.

\section{Soft agar colony formation}

The clonogenic potential of the GSC line was assessed by soft agar assay wherein, $10^{4}$ cells per well were seeded in 6-well plates, in a $0.3 \%$ soft agar solution with $10 \%$ FBS containing complete medium, on top of a $1 \%$ agarose basal layer. The culture medium was also added to soft agar and refreshed every 3-4 days for 3 weeks. The cells were then fixed in $4 \%$ formaldehyde and stained with crystal violet for colony counting.

\section{Generation of stable NOTCH1 and WNT5A knockdown cells}

Three individual shRNAs to NOTCH1 and WNT5A were designed using the RNAi Consortium shRNA library, BROAD institute, Cambridge, MA, and cloned into pLKO.1-TRC plasmid. lacZ sense and lacZ antisense sequence were used as the non-targeting control shRNA for the experiments. The shRNA plasmid constructs were confirmed by sequencing using human U6 promoter-specific forward primer and were designated as NOTCH1-shRNA1, 2, and 3; WNT5A-shRNA1,2 and 3 and shlacZ (Control). The sequences of shRNAs designed for targeting NOTCH1 and WNT5A transcripts and non-targeting Control shRNA are listed in Supplementary Table 2.

The HEK293T cell line was transfected using a $2^{\text {nd }}$ generation lentiviral system for producing lentiviral vectors with Invitrogen Lipofectamine 3000 following manufacturer's protocol. Lentiviral vectors (pMD2.G, Cat\# 12259 and psPAX2, Cat\# 12260) were purchased from Addgene Inc. (U.S.). Knockdown efficiencies of individual shRNA sequences were determined at RNA and protein levels (Supplementary figs. S2 d-i, d-ii, e \& f).

Out of 3 shRNAs used to knock down NOTCH1, we obtained better knock-down efficiency with only shRNA2 in KW as well as NSG-70 GSC (Fig.S2e; Fig.S2f). However, MUR GSCs showed reasonable knockdown efficiency with all the three shRNAs.

\section{Generation of stable WNT5A overexpressing GSC lines}

To generate stable cell lines, lentiviral transduced control GSCs (NSG-70-GFP and KW-GFP) and WNT5A overexpressing GSCs (NSG-70-WNT5A-GFP and KW-WNT5A-GFP) were sorted by flow cytometry 
(FACSAria). Three independent experiments were performed to obtain over expressing GSC lines. Vectors (Sino Biologicals Inc.) used for lentiviral preparation are listed in supplementary information.

\section{Statistical analysis}

All data were obtained from at least three replicates and quantitative data are presented as mean \pm standard deviation. Statistical analysis was performed using GraphPad Prism 5.0 software. Student's $t-$ test was applied to compare differences between two groups and one-way ANOVA was used for multiple comparisons. Statistical significance was denoted as: ${ }^{*} p<0.05,{ }^{* \star} p<0.01,{ }^{* \star *} p<0.001$ and ${ }^{* \star \star} p<0.0001$. All error bars represent the standard deviation of the mean. Unless otherwise mentioned, all experiments were performed at least in triplicate.

\section{Results}

\section{Characterization of NSG-70, a new glioblastoma stem cell line which harbours a marker isochromosome $9 q(i 9 q)$}

NSG-70, a long-term cell line was derived from tumor tissue of a patient with recurrent glioma and has been cultured through more than 25 passages, whereas concurrently established normal brain cell cultures (NB) derived from epilepsy patient-derived biopsies could not be maintained beyond 5- 6 passages. The adherent NSG-70 culture presented as small, translucent, spindle-shaped cells characteristic of Grade IV gliosarcoma tumors[18],[19] that could also generate non-adherent organised neurospheres within 8-10 days in culture at a high frequency (55-60\%), indicating a glioma stem cell (GSC)-like nature (Fig. 1a).

NSG-70 GSCs displayed a low proliferation potential with 150h doubling time (determined through MTT assay and low Ki-67 expression, Figs.S1a;S1b). This slow-cycling (almost quiescent) nature was validated by PKH dye quenching assay that retained the dye in around $98.5 \%$ population even after six days in culture (Fig.S1C), and further reflected in high latency during xenograft formation, with all mice developing small tumors after four months of injection $\left(10^{6}\right.$ cells; Fig.S1d). Immunohistochemistry of the NSG-70 xenografts displayed typical spindle shaped morphology of the tumor cells that ascertained the gliosarcoma phenotype, along with wild type ATRX and IDH1, and mutated p53 expression (Fig. 1b; Fig.S1e). Ki-67 and CD34 expression was restricted to $<40 \%$ cells, affirming its low proliferation index and indicating scanty tumor vessel density respectively.

To ascertain their stem-like nature, we profiled NSG-70 cells for expression of neural progenitor (SOX2, NESTIN and MSI1) as well as differentiation markers (glial fibrillary acidic protein - GFAP and S100 calcium-binding protein - S100 $\beta$ for astrocytic; TUJ1 and MAP2 for neuronal and OLIG2 for oligodendroglial lineages; Fig. 1c-i). Immunostaining of NSG-70 neurospheres also identified enhanced expression of stem cell markers CD133, SOX2, NESTIN and MSI1, while flow cytometry-based profiling and quantification indicated the presence of 13\% CD133 and 69.6\% CD44-positive cells in adherent cultures (Figs. 1c-ii,d-I and d-ii). Presence of serum in the culture medium reduced the levels of stem cell 
marker expression (Fig. 1e). Xenograft tumors also expressed high levels of SOX2, CD44 and GFAP (Fig. 1f), confirming the presence of GSC-like cells along with differentiated cells. Further study of gross chromosomal rearrangements in NSG-70 cells in culture (serum as well as serum-free medium) indicated the cell line to be aneuploid with random loss and gain of various chromosomes in different metaphases, which lead to a near tetraploid complement (Fig. 1g-i). The most striking feature in a large majority of cells in the population was the presence of an isochromosome of the long arm of chromosome 9 (Isochromosome 9q or i9q). Chromosomal or cytogenomic microarray analysis that involves differential binding of specific probes to the site of genomic imbalances to enable identification of deletions/loss, duplications (gain/amplification), unbalanced translocations validated the presence of isochromosome 9q, with specific gain of cytoband q21.11-q34.3 (Fig. 1g-ii). Strikingly, this location harbors the NOTCH1 gene, which is of special interest in the context of GSC regulation and functions.

\section{NSG-70 GSC culture is likely to represent an in vitro model of mixed lineage}

In order to understand the molecular networks regulating NSG-70 cells, we profiled their gene expression along with two other GSC cell lines earlier established in the lab (KW and MUR) and further compared them with the expression of NB primary cultures. This profiling indicated significant alteration of specific genes and pathways in GSCs over normal, suggesting their possible contribution to gliomagenesis (Fig.S2a).

We hence oriented our study to compare the levels of stem cell and neural lineage markers in the GSC lines relative to hNSC and NB cells. All cells expressed considerable heterogeneity of these markers although high levels of CD44 expression in the 3 GSCs and lower levels of stem cell markers in NB cells were observed (NESTIN, OCT4 and NANOG; Fig. 2a; Fig.S2b) possibly indicating their roles in maintaining progenitor pool and multipotency. Significantly higher expression of oligodendrocyte lineage transcription factor 2 (OLIG2), neuronal precursor marker doublecortin (DCX), astrocytic marker (GFAP) was identified in GSCs compared to NB, the latter suggests astrocytes to be the predominant population (Fig. 2b). OLIG2 expression was highest in NSG-70 cells, suggesting that a subset of cells is committed to an oligodendroglial fate towards a proneural phenotype. The heterogeneity in the expression of stem cell and lineage markers was also reflected at the protein level (Figs. 2c; Fig.S2c).

Further, to delineate the subtype of GSC cultures, we profiled the expression levels of proneural and mesenchymal subtype-specific genes in the 3 GSC cell lines, which revealed KW to be predominantly proneural and MUR as mesenchymal, while NSG-70 presents with a mixed lineage (proneural and mesenchymal subtypes (Fig. 2d). In conclusion, NSG-70 is a new primary glioblastoma cell line representing a mixed (proneural and mesenchymal) subtype with high expression level of self-renewal molecules indicating its inherent stem-like nature with potential to differentiate into different lineages, along with a striking feature of a novel marker i9p marker.

\section{NOTCH1 knockdown leads to reduction in stemness and mesenchymal markers, along with decreasing WNT5A expression}


To elucidate the role of Notch pathway in self-renewal and differentiation of stem cells, we first evaluated the effects of NOTCH1 knockdown on stemness in the three GSC lines (NSG-70, KW and MUR). Our results demonstrated diminished expression of stemness as well as mesenchymal markers such as CD44, TWIST1 and FN1 in the 3 GSC lines (Figs. 2h-i; 2h-ii; 2i; Figs. S2g-i; 2g-ii; 2h). The cross-talk of Notch 1 and Wnt signaling pathway has been shown as crucial in stem cell maintenance (Collu et al. 2014). However, recent reports suggested the role of Wnt5a in inducing mesenchymal-like characteristic in GSCs[13], there is a dearth of knowledge on the cross-talk of NOTCH1 and WNT5A. Hence, we sought to understand the significance of these signaling pathways in glioma. We identified significantly higher expression of NOTCH1 and WNT5A mRNA in primary GBM tumor tissues compared to normal tissues in the UALCAN database (Figs. 2e-i; 2e-ii). Further examining the expression levels of these two molecules in the three GSC lines, we observed that MUR cells expressed highest levels of both NOTCH1 and WNT5A, whereas KW and NSG-70 expressed moderate levels of NOTCH1 and very low levels of WNT5A (Figs. 2f; 2g). Interestingly, knockdown of WNT5A alone in WNT5A-high cells i.e., MUR also showed significantly decreased levels of stem cell and mesenchymal markers (Figs. 3a; 3b), suggesting coordinated action of NOTCH1 and WNT5A in maintaining stemness and promoting mesenchymal characteristics in GSCs.

\section{WNT5A overexpression or induction in GSCs enhances NOTCH1 levels, stem cell and mesenchymal marker expression}

In either case of NOTCH1 and WNT5A knockdown, reduction in the levels of stemness and mesenchymal markers at RNA and protein levels were concurrently detected in GSC lines; this suggests a coregulation of $\mathrm{NOTCH} 1$ and WNT5A in GSCs. Prompted by this observation, we assessed NOTCH1 expression upon overexpression of WNT5A in NSG-70 and KW (WNT5A-low GSCs) at RNA and protein levels, to explore if WNT5A wcould influence NOTCH1 levels. The data indicated elevated expression of NOTCH1 and its target gene HES1, stem markers (NESTIN, SOX2), and mesenchymal markers (FN1, TWIST1, VIMENTIN) in WNT5A overexpressed GSCs compared to their control counterparts (Figs. 3c; 3d). This suggests that components of WNT5A pathway synergize with NOTCH1, promoting GSC characteristics and thereby contributing to tumor progression.

\section{WNT5A knockdown drives GSCs into proliferative phase}

We also detected lower levels of p21 upon NOTCH1 knockdown in NSG-70 and MUR (Figs. 2h-ii ; 2i) whereas higher p21 levels after WNT5A overexpression (Fig. 3c), which indicated the involvement of WNT5A in cellular proliferation.

We further assessed the cell cycle progression of MUR (WNT5A silenced) as well as NSG-70 and KW (WNT5A overexpressed or rhWNT5A induced). Our data demonstrated that WNT5A knockdown in MUR facilitates cell proliferation marked by an increase in S phase cells (Fig. 3Sf). Flow cytometry and immunofluorescence to evaluate Ki67 positive population, along with MTT assay in MUR, also displayed similar results (Figs. 3e, f and g). All these data suggested that WNT5A knockdown drives GSCs into proliferative phase. Contrarily, the reduction in cellular proliferation upon WNT5A overexpression in WNT5A-low GSCs (NSG-70 and KW) as indicated by flow cytometry to measure the Ki67 positive 
population as well as MTT assay (Figs. 3h, i-i, and i-ii), supports the role of WNT5A in maintaining GSC quiescence.

\section{NOTCH1 in association with WNT5A, promotes vasculogenic mimicry in GSCs besides maintaining stem cell vigor}

The decreased levels of VEGFA and tumorigenicity observed in KW cells following NOTCH1 knockdown (Figs. 4a;4b; Fig. S3a), along with NOTCH1 being an important regulator of vasculogenic mimicry (Fig. $4 d$ ), we were curious to evaluate whether rhWNT5A too would have promoting vasculogenic mimicry in the WNT5A-low GSC (NSG-70) and on comparison with WNT5A-high GSCs (MUR). Our data showed almost $50 \%$ increase in the total number of junctions as well as the tube length in rhWNT5A treated cells compared to control. NOTCH1 knockdown significantly decreased both these parameters by approximately $75 \%$ whereas rhWNT5A treatment of NOTCH1 silenced cells effectively rescued the ability to form vessel like structures (Fig. 4d). Additionally, WNT5A induction of GSCs also resulted in higher expression of not only NOTCH1 but also other angiogenesis related genes including CD31, VEGFA and PDGFRa (Fig. 4C). This underlines the involvement of WNT5A in promoting vasculogenic mimicry in GSCs and might represent one of the crucial alternative ways by which GSCs promote tumor neovascularization.

Further to investigate whether silencing NOTCH1 and WNT5A expression was associated with reduced stem cell vigor of GSCs, we analysed the surface expression of the GSC marker CD44, by flow cytometry. NOTCH1 knockdown showed significant reduction in the CD44 expression in KW and MUR, while nonsignificant reduction in NSG-70 (Figs. 4e-i; 4e-ii, 4e-iii). WNT5A knockdown in MUR also showed decrease in CD44 levels (Fig. 4e-iv). Further to compare the frequencies of stem cells in control and NOTCH1 knockdown MUR cells, we compared their neurosphere formation ability and observed a significant reduction in the number as well as size of neurospheres generated from single cells (Fig. 4f). Similarly, we also observed a remarkable decrease in the adherent independent tumor cell growth and invasive ability of MUR upon NOTCH1 as well as WNT5A knockdown, as represented by the soft agar assay (Fig. 4g). All this together implies the collective role of NOTCH1 and WNT5A in maintaining stem cell characteristics in GSCs.

\section{Discussion}

GBM tumors are extremely heterogeneous with numerous reports supporting the likely involvement of GSCs in the maintenance of cellular heterogeneity. GSCs play a crucial role in the emergence and progression of GBM tumors, substantiating GSCs as a key target for treatment. Accumulating evidence emphasizes on the several developmental signaling pathways such as the Notch, Wnt and Sonic hedgehog $(\mathrm{SHH})$ in tweaking the differentiation and proliferation of GSCs, along with modulating tumor invasion and neoangiogenesis[8]. Hence, in the current study, we first established and characterized a GSC culture, referred to as NSG-70 from recurrent grade IV glioma patient-derived tumor tissue to comprehend the role of GSCs in tumor sustenance. Since the characteristic property of stem cells is their 
ability to differentiate into various cell types, we examined the stem cell characteristics of NSG-70 GSCs by analysing the expression of stem cell markers such as CD133, SOX2, NESTIN, and MSI1 along with differentiation markers such as GFAP, $S 100 \beta$, TUJ1, and MAP2 by qPCR and immunofluorescence. The data showed positivity for all the stem cell markers with a very few TUJ1 + and MAP2 + neural like cells, confirming the stem-like feature of NSG-70 culture. As GSCs are also reported as the quiescent population resistant to treatments that preferentially target rapidly dividing cells, we performed IF staining for Ki67, MTT and PKH dye dilution assays to check the proliferative status of NSG-70 GSCs. The data showed only few positive cells in Ki67 staining and extremely slow dilution of the PKH dye, collectively suggesting the slow dividing nature of the NSG-70 culture.

Regarding the origin of GSCs, there are ambiguous beliefs with some group supporting the idea that GSCs arise from developmentally arrested neural progenitors while others proposing that they emerge from dedifferentiated astrocytes[20]. Sandberg et al. in 2013 showed that GSCs and adult human NSCs (ahNSCs) share similar levels of stemness and neural lineage markers, however, developmental pathway genes are significantly upregulated in GSCs compared to ahNSCs[21]. We used iPSC-derived hNSCs to compare the expression pattern of stem cell as well as neural markers in NSG-70 with respect to hNSCs. Here, we utilised two other previously established grade IV GSC cultures (KW and MUR) for the comparison. In our study, we observed very heterogeneous expression pattern for all these markers among the 3 GSC cultures with respect to hNSC except for remarkably high expression of CD44 and GABRA1 in GSC cultures compared to hNSC. It might suggest the inherent heterogeneity among the GSC cultures specifying the need to assign tumor subtype to these cultures based on predominant transcriptional profiles and specific genetic alterations as described by Verhaak et al. 2010. GSC subtyping will ease the complexities involved in study to use them as a model for personalized therapy and achieving better prognosis. Verhaak et al. categorized GBM tumors into 4 distinct molecular subtypes- classic, neural, proneural and mesenchymal[4]. However, Lottaz et al. 2010 proposed only 2 subgroups of GBM-proneural and mesenchymal which possesses the transcriptional profile of fetal NSC (fNSC) and adult NSC (aNSC) respectively. This subgrouping highlights the different origin and activation of different signaling pathways in proneural and mesenchymal tumors[22]. Here, we profiled the 3 GSCs along with hNSC and we found KW as proneural, MUR as mesenchymal while NSG-70 as a mix of proneural and mesenchymal subtype.

Since, all tumors with IDH mutation are essentially secondary glioblastomas which reflect proneural subtype expression, we determined the mutation status of IDH1 by staining for mutated-IDH1 along with mutated p53 and wild type ATRX using IHC. NSG-70 xenograft sections were negative for IDH1 mutation and showed ATRX retention, confirming NSG-70 as a primary tumor, which could belong to virtually any subtype. KW xenografts on the other hand, showed IDH1 mutation and ATRX loss, indicating KW tumor as secondary glioblastoma of proneural subtype. Additionally, glioblastomas are cytogenetically heterogeneous tumor. Hence, we determined the various chromosomal alterations in NSG-70 GSC line by karyotype analysis which revealed an unique cytogenomic feature, i.e., isochromosome $9 q$ (i9q) along with several previously reported chromosomal aberrations. We, then validated the isochromosome $9 q$ presence by chromosome microarray analysis, which also illustrated a specific gain of cytoband q21.11- 
q34.3, harboring the NOTCH1 gene. This finding corroborated with the accumulating evidence suggesting the crucial role of Notch signaling in the NSCs and its deregulation in GBM[23], wherein activated Notch signaling is correlated with the stem-like phenotype of GSCs[24].

Notch pathway has been demonstrated to promote proliferation and suppress differentiation of neural precursor cells[25]. Hence, we first investigated the effect of NOTCH1 on GSC stemness. Our results demonstrated that NOTCH1 knockdown significantly inhibited the stemness markers such as NESTIN and SOX2 as well as mesenchymal markers- CD44 and TWIST1, along with concomitant downregulation of WNT5A. Recent reports demonstrated WNT5A to promote epithelial-mesenchymal transition, migration and invasion in non-small-cell lung cancer and pancreatic cancer[26],[27]. In GBM tissues also, we found significantly higher mRNA expression of NOTCH1 and WNT5A compared to normal brain according to TCGA data based UALCAN database. This observation was supported by the high RNA and protein levels of NOTCH1 in all the $3 \mathrm{GSCs}$, however they showed very different levels of WNT5A. WNT5A was expressed highly in mesenchymal subtype of GSC (MUR) whereas in proneural (KW) and proneuralmesenchymal mix (NSG-70) GSCs, WNT5A levels were low at RNA and protein levels. Given the fact that NSG-70 line was recurrent GBM-derived line, phenotypic plasticity of GSCs wherein proneural to mesenchymal subtype transition or vice-versa occurs, resulting in therapy resistance and tumor recurrence[28], might be the cause for mixed subtype in NSG-70. It also signifies the subtype-specific expression of WNT5A in GSCs. This is in line with the previous studies showing WNT5A overexpression induces an infiltrative, mesenchymal-like characteristics in high-grade gliomas[13].

Notch and Wnt/ $\beta$-catenin pathways modulate motility and invasion by activating an epithelialmesenchymal like program in GBM[7]. However, any elaborate study showing the role of non-canonical WNT5A in the regulation of the maintenance and proliferation of GSCs is lacking. Hence, we next examined the effect of WNT5A downregulation in MUR (WNT5A-high GSC) and we found a remarkable reduction in stem cell as well as mesenchymal markers, along with NOTCH1 inhibition at RNA and protein levels.

One of the hallmarks of GBM are rapid infiltrating growth and extensive vasculature. Several reports elicited induction of VEGFA and Notch signaling genes, as an angiogenic response to hypoxia. Our data showed reduced VEGFA level and vasculogenic mimicry in GSCs (NSG-70 and MUR) upon NOTCH1 knockdown. Consistent with the previous findings that Notch inhibition leads to the reduced tumorigenicity of GSCs [29], we observed reduced tumor volume with very less vascularity in NOTCH1 knockdown GSC injected mice compared to that of control. This suggests that NOTCH1 might play a role in promoting tumor angiogenesis.

Moreover, the role of Wnt5a in inducing endothelial cell proliferation and survival under serum-deprived conditions had been documented earlier[30]. Recently, GSC differentiation into endothelial-like cells (GdECs) mediated by WNT5A was shown by Hu B et al., 2017. However, there are no studies showing the involvement of WNT5A in vasculogenic mimicry of GSCs to date in glioma or the coordination of WNT5A and NOTCH1 in GSC maintenance. Our results showed that WNT5A knockdown lowers down the 
expression of angiogenic molecules as well as vasculogenic mimicry in GSCs. On the contrary, upon WNT5A overexpression or rhWNT5A induction of GSCs, we noted an increase in the levels of angiogenic molecules (Fig. 3b and d) as well as vessel branches representative of vasculogenic mimicry. Together, our results reveal the collective role of NOTCH1 and WNT5A signaling in tumor angiogenesis.

Current work elucidates the coordinated action of NOTCH1 and WNT5A signaling pathways in GSC biology. The work focuses on improving the understanding of GSC self-renewal and maintenance and how NOTCH1 and WNT5A signaling are closely associated with modulating tumor angiogenesis. From this work, it seems clear that the effects of NOTCH1 and WNT5A are in sync, but further investigation is required to shed light on whether the coinciding effect of the two pathways is mediated by NOTCH1 itself or downstream effectors of the pathway. However, both the molecules cooperate to modulate stem cell self-renewal, proliferation, and angiogenesis in GSCs (Fig. 5). We clearly believe that the NOTCH1-WNT5A axis could be harnessed in future as a promising link to develop effective GSC-directed therapies in GBM.

In conclusion, NOTCH1 and WNT5A signaling pathways work synergistically and regulate GSCs selfrenewal, proliferation, and invasion, and vasculogenic mimicry to aid tumor neoangiogenesis. NOTCH1WNT5A axis needs to be further explored, which could have great clinical implications and could yield promising therapeutic targets for GBM.

\section{Abbreviations}

\begin{tabular}{ll} 
GBM & Glioblastoma multiforme \\
\hline CNS & Central Nervous System \\
\hline GSC & Glioma stem cell \\
\hline TCGA & The Cancer Genome Atlas \\
\hline bFGF & Basic fibroblast growth factor \\
\hline EGF & Epidermal growth factor \\
\hline NOD-SCID & Nonobese diabetic/severe combined immunodeficiency \\
\hline GFAP & Glial fibrillary acidic protein
\end{tabular}

\section{Declarations}

\section{Acknowledgements}

We acknowledge the core facilities like animal house, confocal imaging and FACS facility of National Centre for Cell Science (NCCS), Pune, India for the technical assistance and support required to perform the experiments.

\section{Author Contributions}

Page 13/26 
SAB and AnS conceptualized and planned the study design, DKS performed, analysed and interpreted experimental data and wrote the manuscript. $S A B$ interpreted experiments and edited the manuscript draft to its final form. PKMS established iPSC-derived hNSC culture, designed shRNA constructs for NOTCH1 gene and lentiviral preparations for WNT5A overexpression constructs.AmS and AM established and provided GSC culture models (KW and MUR) and helped with evaluation, DM provided GBM patient tumor tissues.

\section{Funding}

This work was supported by the Department of Biotechnology, Government of India (Project noBT/PR10852/MED/30/1315/2014) and the National Centre for Cell Science (NCCS) Intramural funds. DKS was granted junior and senior research fellowship by Department of Biotechnology, India. PKMS was granted junior and senior research fellowship by University Grants Commission, India. Fellowship support to AmS-Council of Scientific and Industrial Research (CSIR) New Delhi; AM was supported by fellowship provided by Department of Biotechnology, New Delhi, India (Project noBT/PR10852/MED/30/1315/2014).

\section{Conflicts of Interest}

The authors declare no conflict of interest.

\section{References}

1. Singh SK, Hawkins C, Clarke ID, Squire JA, Bayani J, Hide T, Henkelman RM, Cusimano MD, Dirks PB (2004) 4327015 Identification of human brain tumour initiating cells, Nat. 432 (2004) 396-401. https://doi.org/10.1038/nature03128

2. Visvader JE, Lindeman GJ (2008) Cancer stem cells in solid tumours: accumulating evidence and unresolved questions. Nat Rev Cancer 8:755-768. https://doi.org/10.1038/NRC2499

3. Qiang L, Wu T, Zhang HW, Lu N, Hu R, Wang YJ, Zhao L, Chen FH, Wang XT, You QD, Guo QL (2012) 192 HIF-1a is critical for hypoxia-mediated maintenance of glioblastoma stem cells by activating Notch signaling pathway, Cell Death Differ. 19 (2011) 284-294. https://doi.org/10.1038/cdd.2011.95

4. Verhaak RGW, Hoadley KA, Purdom E, Wang V, Qi Y, Wilkerson MD, Miller CR, Ding L, Golub T, Mesirov JP, Alexe G, Lawrence M, O'Kelly M, Tamayo P, Weir BA, Gabriel S, Winckler W, Gupta S, Jakkula L, Feiler HS, Hodgson JG, James CD, Sarkaria JN, Brennan C, Kahn A, Spellman PT, Wilson RK, Speed TP, Gray JW, Meyerson M, Getz G, Perou CM, Hayes DN (2010) Integrated Genomic Analysis Identifies Clinically Relevant Subtypes of Glioblastoma Characterized by Abnormalities in PDGFRA, IDH1, EGFR, and NF1, Cancer Cell. 17:98-110. https://doi.org/10.1016/J.CCR.2009.12.020

5. Safa AR, Saadatzadeh MR, Cohen-Gadol AA, Pollok KE, Bijangi-Vishehsaraei K (2015) Glioblastoma stem cells (GSCs) epigenetic plasticity and interconversion between differentiated non-GSCs and GSCs. Genes Dis 2:152-163. https://doi.org/10.1016/J.GENDIS.2015.02.001 
6. Mao P, Joshi K, Li J, Kim SH, Li P, Santana-Santos L, Luthra S, Chandran UR, Benos PV, Smith L, Wang M, Hu B, Cheng SY, Sobol RW, Nakano I (2013) Mesenchymal glioma stem cells are maintained by activated glycolytic metabolism involving aldehyde dehydrogenase 1A3, Proc. Natl. Acad. Sci. U. S. A. $1108644-8649$.

https://doi.org/10.1073/PNAS.1221478110/-/DCSUPPLEMENTAL

7. Kahlert UD, Maciaczyk D, Doostkam S, Orr BA, Simons B, Bogiel T, Reithmeier T, Prinz M, Schubert J, Niedermann G, Brabletz T, Eberhart CG, Nikkhah G, Maciaczyk J (2012) Activation of canonical WNT/ $\beta$-catenin signaling enhances in vitro motility of glioblastoma cells by activation of ZEB1 and other activators of epithelial-to-mesenchymal transition. Cancer Lett 325:42-53.

https://doi.org/10.1016/J.CANLET.2012.05.024

8. Sharifzad F, Ghavami S, Mardpour S, Mollapour M, Azizi Z, Taghikhani A, Łos MJ, Verdi J, Fakharian E, Ebrahimi M, Hamidieh AA (2019) Glioblastoma cancer stem cell biology: Potential theranostic targets. Drug Resist Updat 42:35-45. https://doi.org/10.1016/J.DRUP.2018.03.003

9. Hu YY, Zheng MH, Cheng G, Li L, Liang L, Gao F, Wei YN, Fu LA, Han H (2011) Notch signaling contributes to the maintenance of both normal neural stem cells and patient-derived glioma stem cells. BMC Cancer 11. https://doi.org/10.1186/1471-2407-11-82

10. Sunayama J, Matsuda KI, Sato A, Tachibana K, Suzuki K, Narita Y, Shibui S, Sakurada K, Kayama T, Tomiyama A, Kitanaka C (2010) Crosstalk between the PI3K/mTOR and MEK/ERK pathways involved in the maintenance of self-renewal and tumorigenicity of glioblastoma stem-like cells. Stem Cells 28:1930-1939. https://doi.org/10.1002/STEM.521

11. Moore KA, Lemischka IR (2006) Stem cells and their niches. Science 311:1880-1885. https://doi.org/10.1126/SCIENCE.1110542

12. Clevers H, Loh KM, Nusse R (2014) Stem cell signaling. An integral program for tissue renewal and regeneration: Wnt signaling and stem cell control. Science 346.

https://doi.org/10.1126/SCIENCE.1248012

13. Binda E, Visioli A, Giani F, Trivieri N, Palumbo O, Restelli S, Dezi F, Mazza T, Fusilli C, Legnani F, Carella M, Di Meco F, Duggal R, Vescovi AL (2017) Wnt5a Drives an Invasive Phenotype in Human Glioblastoma Stem-like Cells. Cancer Res 77:996-1007. https://doi.org/10.1158/0008-5472.CAN-161693

14. Vik-Mo EO, Sandberg C, Olstorn H, Varghese M, Brandal P, Ramm-Pettersen J, Murrell W, Langmoen IA (2010) Brain tumor stem cells maintain overall phenotype and tumorigenicity after in vitro culturing in serum-free conditions. Neuro Oncol 12:1220. https://doi.org/10.1093/NEUONC/NOQ102

15. Sun Y, Pollard S, Conti L, Toselli M, Biella G, Parkin G, Willatt L, Falk A, Cattaneo E, Smith A (2008) Long-term tripotent differentiation capacity of human neural stem (NS) cells in adherent culture. Mol Cell Neurosci 38:245-258. https://doi.org/10.1016/J.MCN.2008.02.014

16. Rathod SS, Rani SB, Khan M, Muzumdar D, Shiras A (2014) Tumor suppressive miRNA-34a suppresses cell proliferation and tumor growth of glioma stem cells by targeting Akt and Wnt signaling pathways. FEBS Open Bio 4:485-495. https://doi.org/10.1016/J.FOB.2014.05.002 
17. Wakimoto H, Mohapatra G, Kanai R, Curry WT, Yip S, Nitta M, Patel AP, Barnard ZR, StemmerRachamimov AO, Louis DN, Martuza RL, Rabkin SD (2012) Maintenance of primary tumor phenotype and genotype in glioblastoma stem cells. Neuro Oncol 14:132-144. https://doi.org/10.1093/NEUONC/NOR195

18. Rutka JT, Giblin JR, Høifødt HK, Dougherty DV, Bell CW, McCulloch JR, Davis RL, Wilson CB, Rosenblum ML (1986) Establishment and Characterization of a Cell Line from a Human Gliosarcoma,Cancer Res.46

19. Kiang KMY, Chan AA, Leung GKK (2021) Secondary gliosarcoma: the clinicopathological features and the development of a patient-derived xenograft model of gliosarcoma. BMC Cancer 21:1-8. https://doi.org/10.1186/S12885-021-08008-Y/FIGURES/4

20. Stiles CD, Rowitch DH (2008) Glioma Stem Cells: A Midterm Exam. Neuron 58:832-846. https://doi.org/10.1016/J.NEURON.2008.05.031

21. Sandberg CJ, Altschuler G, Jeong J, Strømme KK, Stangeland B, Murrell W, Grasmo-Wendler UH, Myklebost O, Helseth E, Vik-Mo EO, Hide W, Langmoen IA (2013) Comparison of glioma stem cells to neural stem cells from the adult human brain identifies dysregulated Wnt- signaling and a fingerprint associated with clinical outcome, Exp. Cell Res 319:2230-2243.

https://doi.org/10.1016/J.YEXCR.2013.06.004

22. Lottaz C, Beier D, Meyer K, Kumar P, Hermann A, Schwarz J, Junker M, Oefner PJ, Bogdahn U, Wischhusen J, Spang R, Storch A, Beier CP (2010) Transcriptional profiles of CD133 + and CD133glioblastoma-derived cancer stem cell lines suggest different cells of origin. Cancer Res 70:20302040. https://doi.org/10.1158/0008-5472.CAN-09-1707

23. Schreck KC, Taylor P, Marchionni L, Gopalakrishnan V, Bar EE, Gaiano N, Eberhart CG (2010) The Notch Target Hes1 Directly Modulates Gli1 Expression and Hedgehog Signaling: A Potential Mechanism of Therapeutic Resistance, Clin. Cancer Res 16:6060. https://doi.org/10.1158/10780432.CCR-10-1624

24. Yu JB, Jiang H, Zhan RY Aberrant Notch signaling in glioblastoma stem cells contributes to tumor recurrence and invasion,Mol. Med. Rep.14 (2016)1263-1268.

https://doi.org/10.3892/MMR.2016.5391

25. Guentchev M, McKay RDG (2006) Notch controls proliferation and differentiation of stem cells in a dose-dependent manner. Eur J Neurosci 23:2289-2296. https://doi.org/10.1111/J.14609568.2006.04766.X

26. Wang B, Tang Z, Gong H, Zhu L, Liu X (2017) Wnt5a promotes epithelial-to-mesenchymal transition and metastasis in non-small-cell lung cancer. Biosci Rep 37:20171092. https://doi.org/10.1042/BSR20171092

27. Bo H, Zhang S, Gao L, Chen Y, Zhang J, Chang X, Zhu M (2013) Upregulation of Wnt5a promotes epithelial-to-mesenchymal transition and metastasis of pancreatic cancer cells. BMC Cancer 13:111. https://doi.org/10.1186/1471-2407-13-496/FIGURES/7 
28. Phillips HS, Kharbanda S, Chen R, Forrest WF, Soriano RH, Wu TD, Misra A, Nigro JM, Colman H, Soroceanu L, Williams PM, Modrusan Z, Feuerstein BG, Aldape K (2006) Molecular subclasses of high-grade glioma predict prognosis, delineate a pattern of disease progression, and resemble stages in neurogenesis. Cancer Cell 9:157-173. https://doi.org/10.1016/J.CCR.2006.02.019

29. Fan X, Khaki L, Zhu TS, Soules ME, Talsma CE, Gul N, Koh C, Zhang J, Li YM, Maciaczyk J, Nikkhah G, DiMeco F, Piccirillo S, Vescovi AL (2010) Eberhart, NOTCH Pathway Blockade Depletes CD133Positive Glioblastoma Cells and Inhibits Growth of Tumor Neurospheres and Xenografts. Stem Cells 28:5. https://doi.org/10.1002/STEM.254

30. Masckauchán TNH, Agalliu D, Vorontchikhina M, Ahn A, Parmalee NL, Li CM, Khoo A, Tycko B, Brown AMC, Kitajewski J (2006) Wnt5a Signaling Induces Proliferation and Survival of Endothelial Cells In Vitro and Expression of MMP-1 and Tie-2. Mol Biol Cell 17:5163. https://doi.org/10.1091/MBC.E0604-0320

\section{Figures}


a
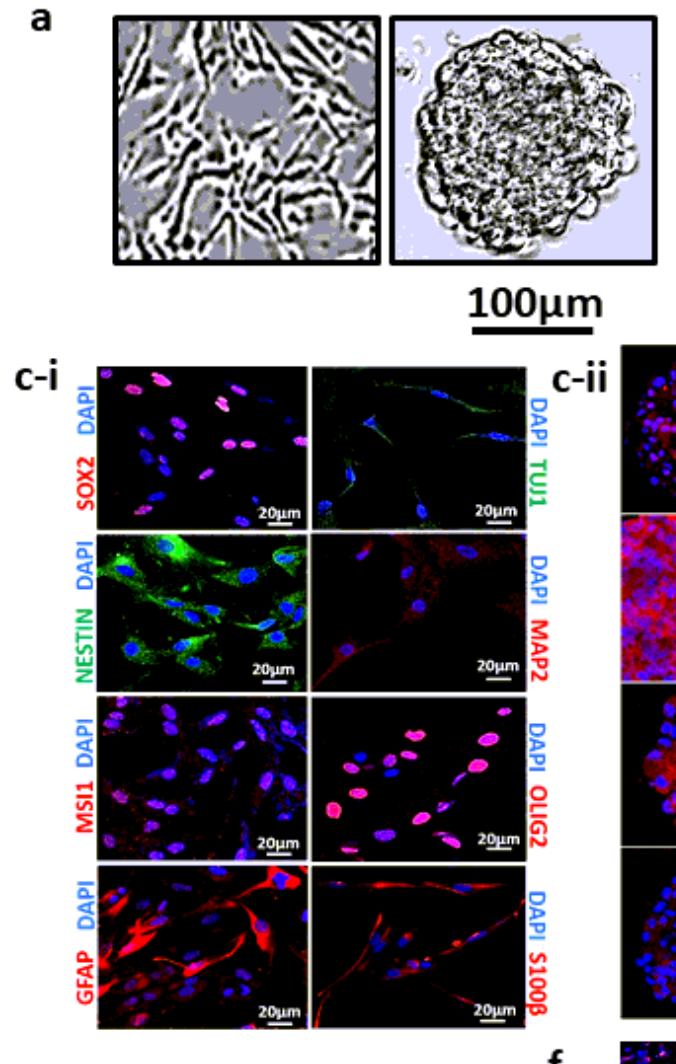

e



g-i

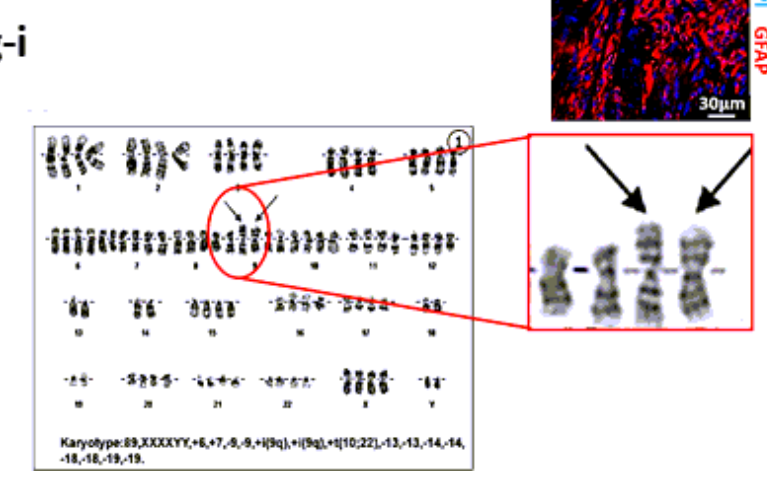

b
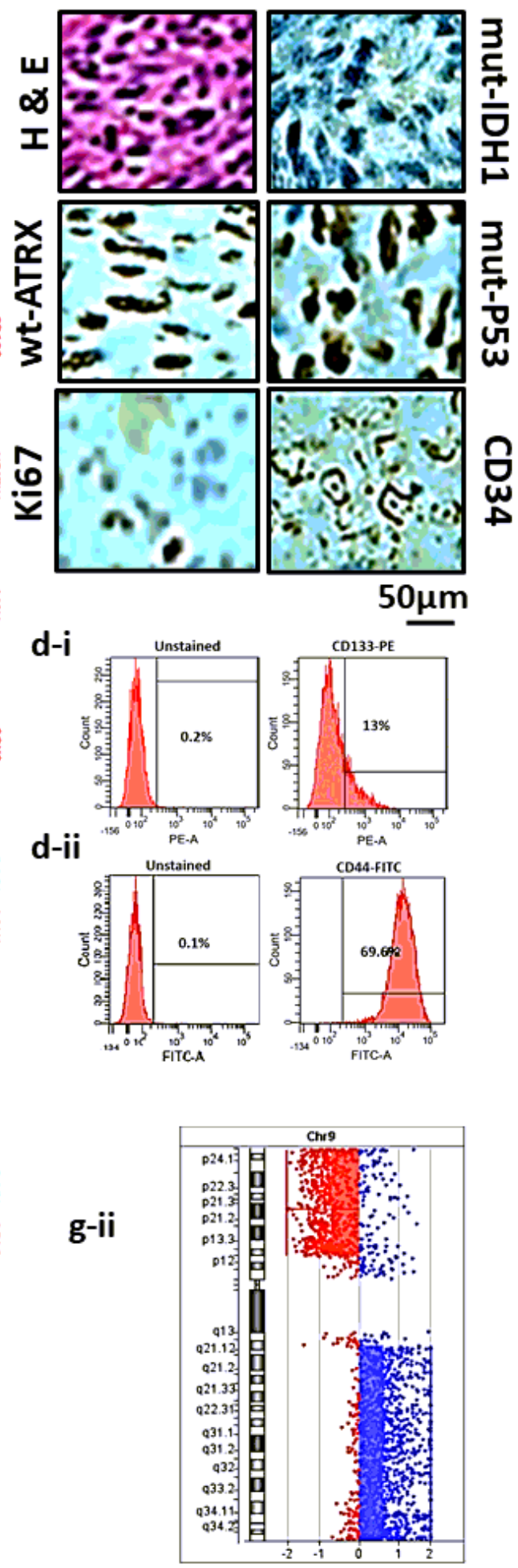

Figure 1

Characterization of a novel GSC line NSG-70. A. Phase contrast images of NSG-70 GSCs cultured either as adherent cells (left) or non-adherent neurospheres derived from single cells (Scale bar: 100 $\mu \mathrm{m}$; magnification: 4X); b. Representative H \& E and immunohistochemistry images of NSG-70 tumor xenograft sections stained for expression of mutated IDH1 (mut-IDH1), wild type ATRX (wt-ATRX), mutated P53 (mut-P53), vessel density marker, CD34 and proliferation marker, Ki67 (Scale bar: 50 4 m; 
magnification: 4X); c. Representative immunofluorescence images indicating expression of, i. astrocytic (GFAP, S100ß), neuronal (TUJ1, MAP2), oligodendrocytic OLIG2) and stem cell (SOX2, NESTIN, MSI1) markers in adherent NSG-70 cultures, ii. stem cell markers (CD133, NESTIN, MSI1 and SOX2) in NSG-70 neurosphere cultures; d. Flow cytometry-based quantification of i. CD133- and ii. CD44-expressing populations in adherent NSG-70 cultures; e. q-PCR analysis to quantify expression of stem cell markers (CD133, NESTIN, SOX2 and CD44) in NSG-70 cells cultured in serum-free and serum containing medium; f. Representative immunohistochemistry images of NSG-70 xenograft sections for SOX2, CD44 and GFAP; g-i. Representative karyotype of NSG-70 cells, arrows indicate isochromosome 9 (i9q); g-ii. Cytogenomic microarray analysis of NSG-70 culture confirming the presence of isochromosome $9 q$. 


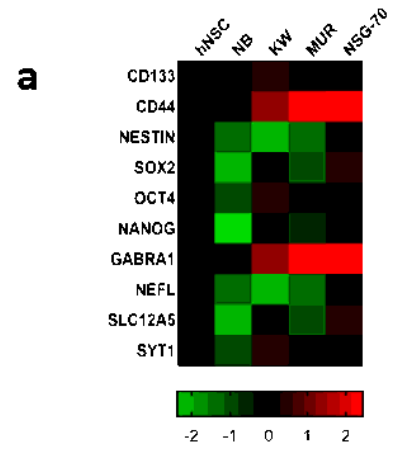

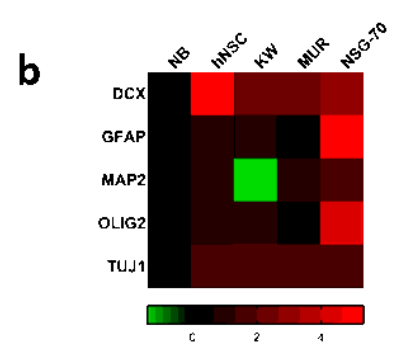

log10 (Relative expression)

\section{log10 (Relative expression)}

d

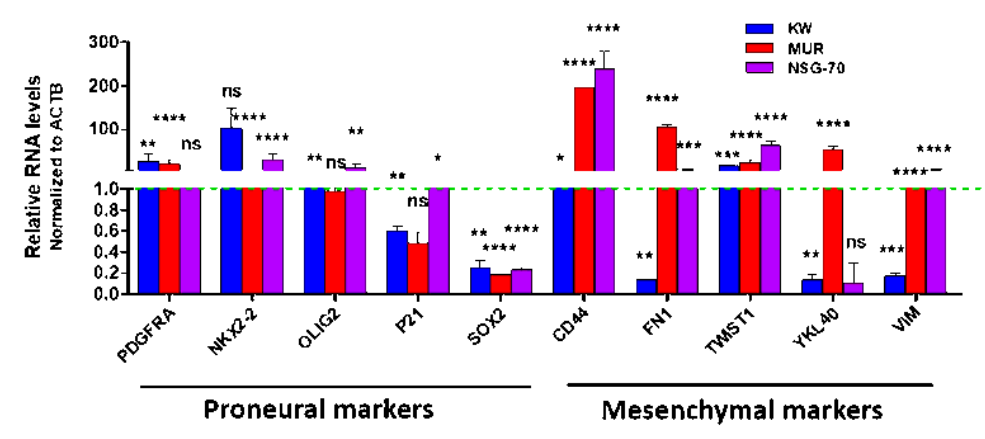

f

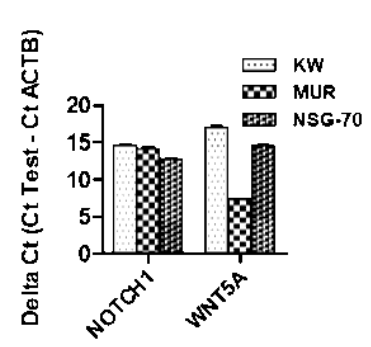

h-i



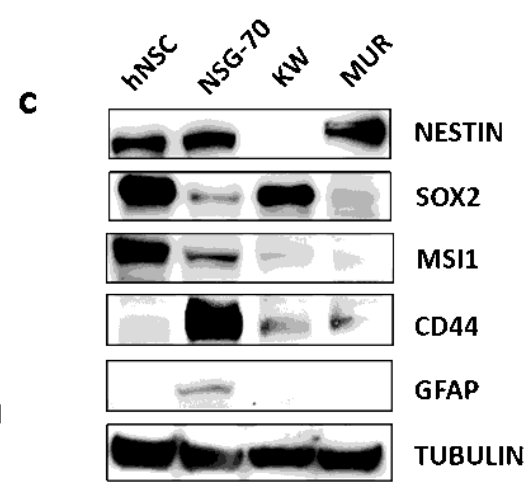

e-i

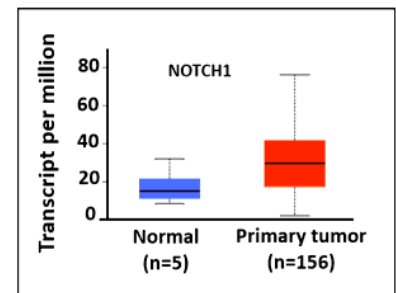

e-ii

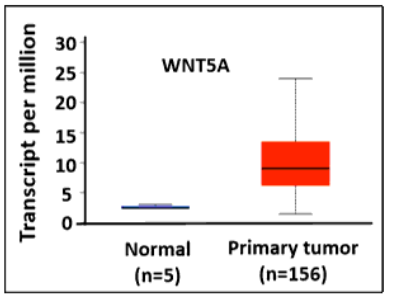

WNTSA TUBULIN

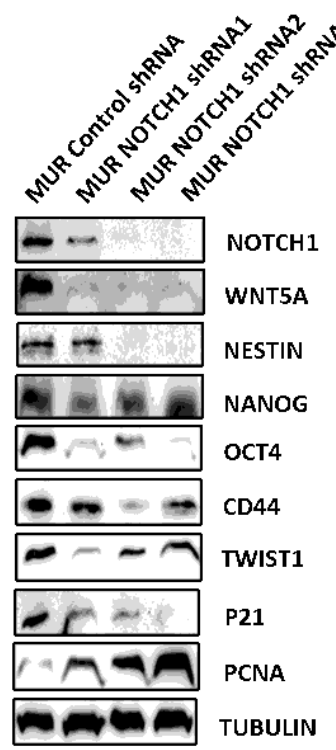

\section{Figure 2}

Heat map representing expression levels of (a) stem cell markers (CD133, CD44, NESTIN, SOX2, OCT4 and NANOG) and neural lineage markers (GABRA1, NEFL, SLC12A5 and SYT1) in the three GSC cultures (KW, MUR and NSG-70) and differentiated glioma cultures (KW10 and MTA10) relative to human neural stem cell (hNSC) culture by qPCR (b) differentiation markers (DCX, GFAP, MAP2, OLIG2 and TUJ1) in the three GSC cultures (KW, MUR and NSG-70) and differentiated glioma cultures (KW10 and MTA10) relative 
to normal brain (NB) culture by qPCR; (c) Expression analysis of stem cell markers (NESTIN, SOX2, MSI1 and CD44) along with astrocytic marker, GFAP in the three GSCs (KW, MUR and NSG-70) and hNSC cultures by western blotting; (d) qPCR showing expression levels of glioblastoma subtype markersproneural (PDGFRA, NKX2-2, OLIG2, P21, and SOX2) and mesenchymal (CD44, FN1, TWIST1, YKL40, and VIM) in GSCs (NSG-70, KW and MUR) calibrated with hNSCs. ACTB was used as the reference gene; (e-i \& e-ii) Expression analysis of NOTCH1 and WNT5A in GBM patients using UALCAN database; Analysis of endogenous level expression of NOTCH1 and WNT5A in 3 GSC cultures (KW, MUR and NSG-70) by (f) qPCR and (g) western blotting; ( $h-i$ \& h-ii) Expression analysis of NOTCH1, HES1, NESTIN, WNT5A, and mesenchymal genes (CD44 and TWIST1) upon stable knockdown of NOTCH1 gene in NSG-70 GSC culture by qPCR and western blotting; (i) Expression analysis of NOTCH1, NESTIN, NANOG, OCT4, and mesenchymal genes (CD44 and TWIST1) upon stable knockdown of NOTCH1 gene by western blotting in MUR GSC culture. 

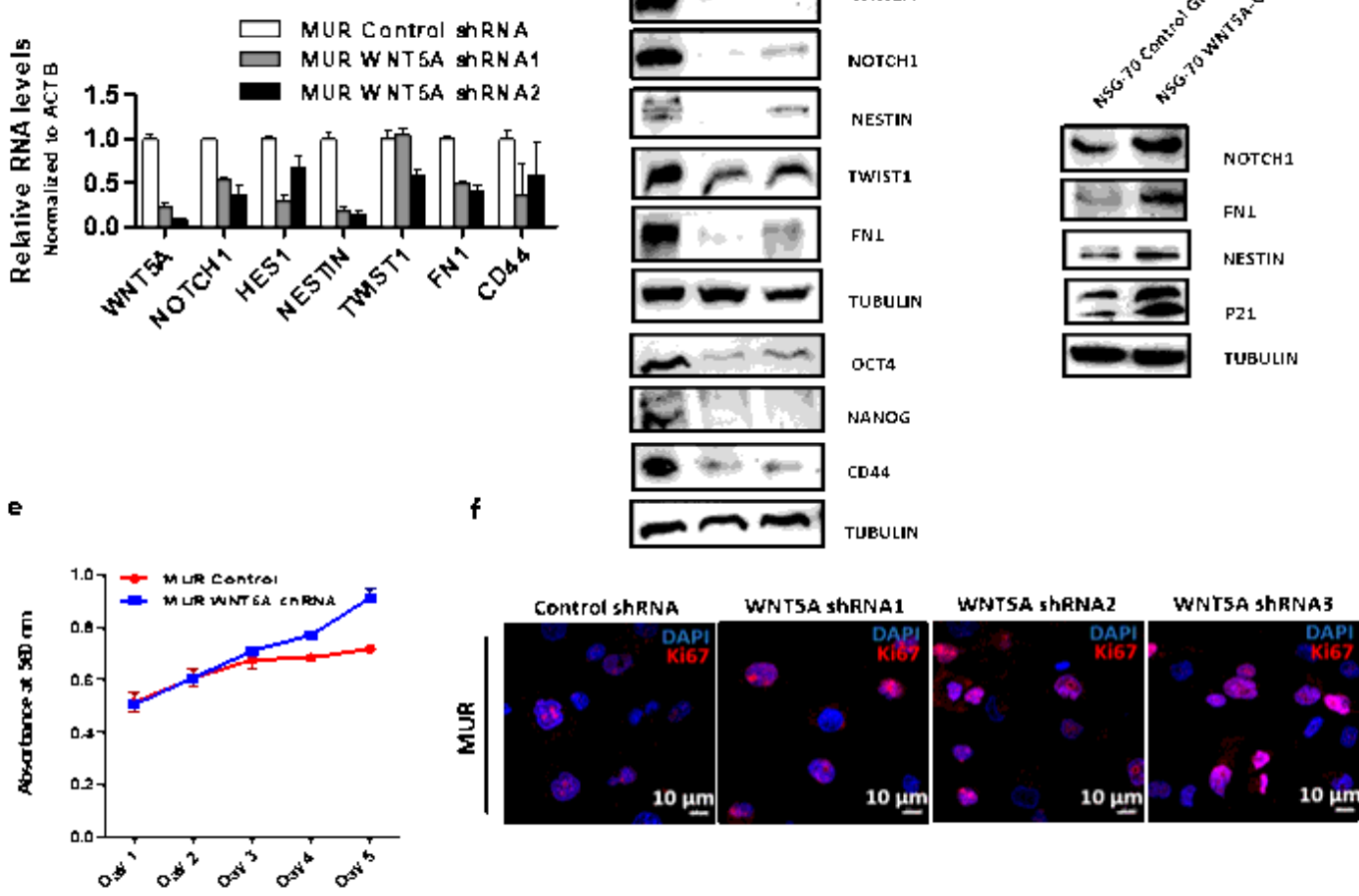

c
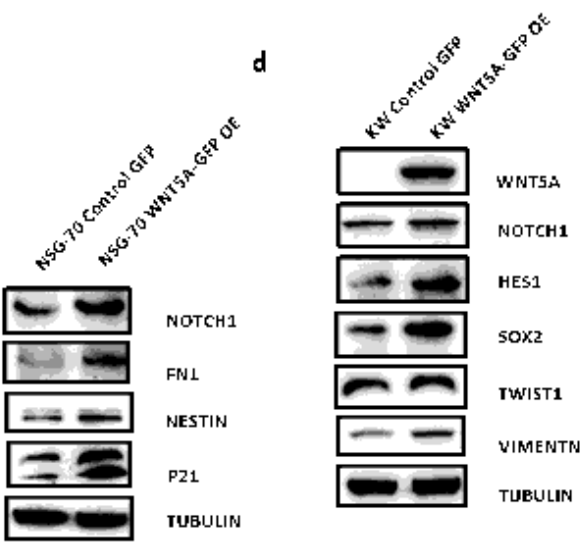

g

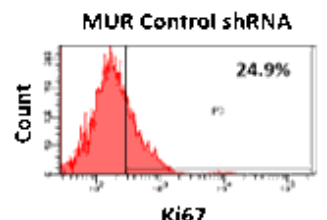

Ki67
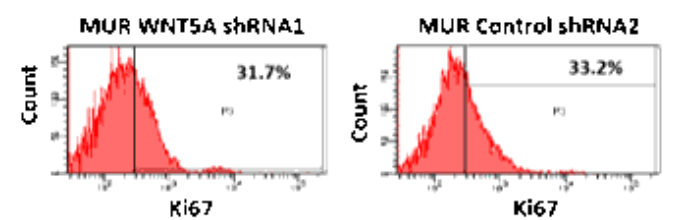

h

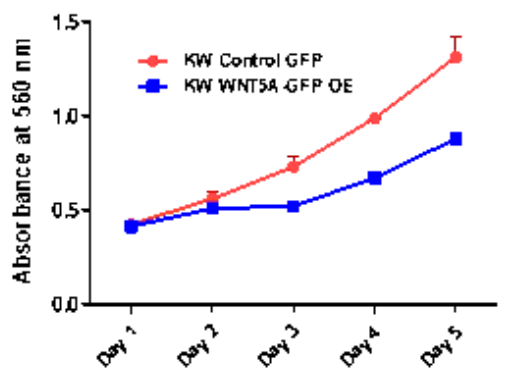

i-i
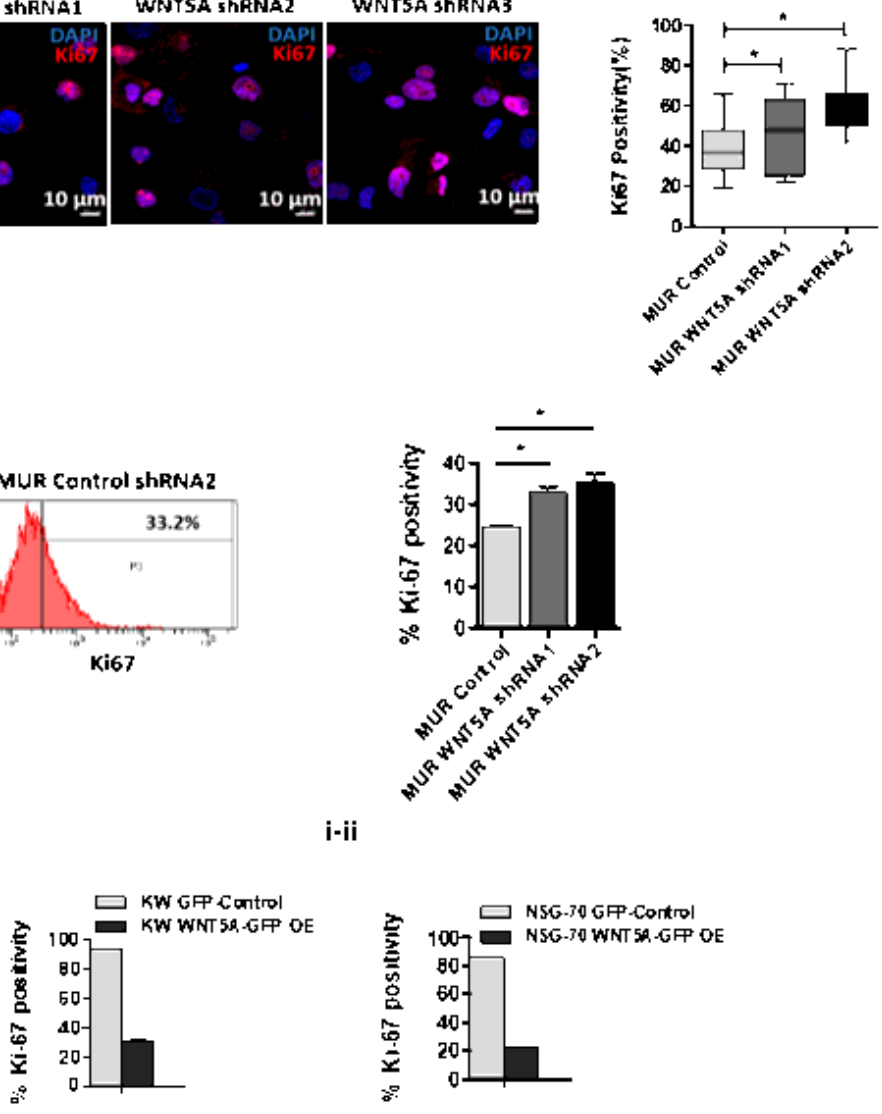

i-ii

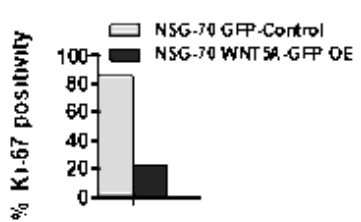

Figure 3

Stable knockdown of WNT5A suppresses stem cell and mesenchymal markers and drives GSCs into proliferative phase (a) qRT-PCR to analyse expression of NOTCH1, NESTIN and mesenchymal markers (CD44, TWIST1 and FN1) upon WNT5A knockdown in MUR (WNT5A-high) GSCs; (b) Western blotting to analyse expression of stem cell (NESTIN, OCT4 and NANOG), and mesenchymal markers (CD44, TWIST1 and FN1) upon WNT5A knockdown in MUR GSCs; Western blotting to analyse expression of stem cell 
and mesenchymal markers upon WNT5A overexpression in WNT5A-low GSCs (c) NSG-70 and (d) KW; (e) MTT assay to determine proliferative ability of MUR GSC upon WNT5A knockdown; (f) Immunofluorescence analysis of Ki67 positive cells in MUR upon WNT5A knockdown, followed by its graphical representation; (g) Flow cytometric analysis of Ki67 positive population in MUR upon WNT5A knockdown, followed by its graphical representation; (h) MTT assay to determine proliferative ability of KW GSC upon WNT5A overexpression; (i) Flow cytometric analysis of Ki67 positive population in WNT5A low-GSCs i. KW, and ii. NSG-70 upon WNT5A overexpression. 

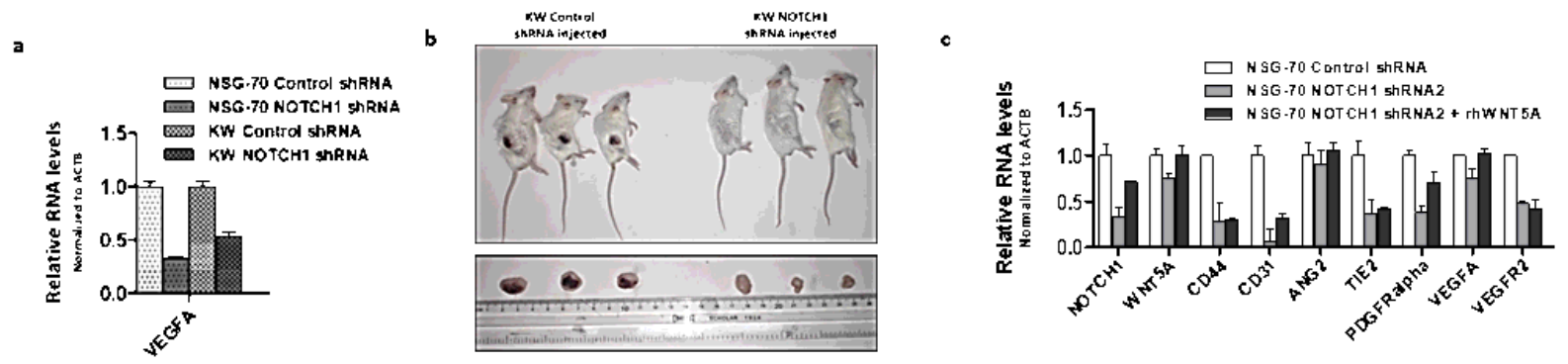

d


e-iii
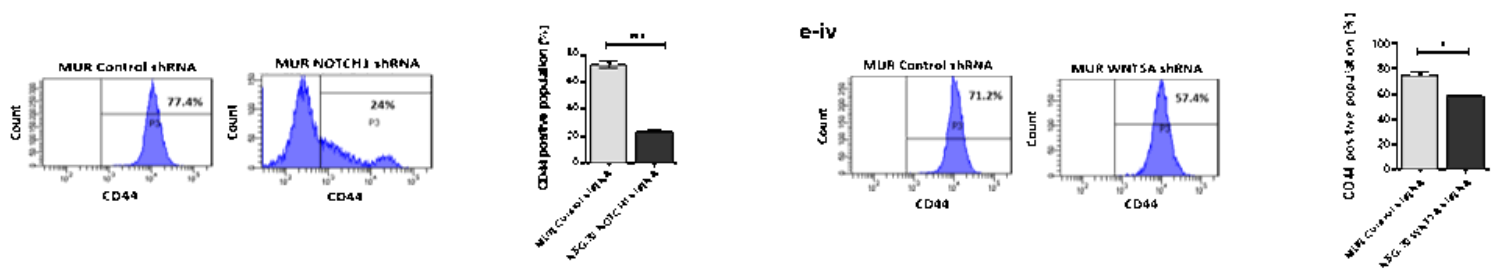

$f$


\section{Figure 4}

(a) qPCR analysis of VEGFA expression in NSG-70 and KW GSC cultures upon stable knockdown of NOTCH1 gene; (b) Control and NOTCH1 knockdown KW GSC-derived tumor in NOD-SCID mice; (c) qPCR analysis of angiogenic gene expression in control and NOTCH1 knockdown NSG-70 upon rhWNT5A induction; (d) Matrigel tube formation assay to assess the effect of rhWNT5A on vasculogenic mimicry using NSG-70 GSCs followed by its graphical representation; (e) Flow cytometric analysis of CD44 
expression in control and NOTCH1 knockdown GSCs i. NSG-70, ii. KW, iii. MUR, and iv. in control and WNT5A knockdown MUR, followed by its graphical representation; (f) Neurosphere formation assay to determine self-renewal potential of control, NOTCH1 and WNT5A knockdown MUR, followed by its graphical representation; (g) Soft agar colony formation assay to determine invasive potential of control, NOTCH1 and WNT5A knockdown MUR, followed by its graphical representation.

\section{Proposed Model}

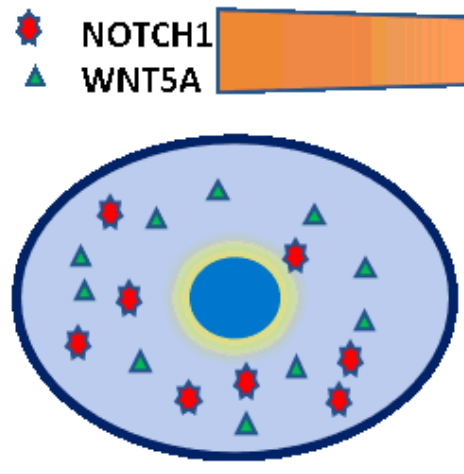

Glioma Stem Cell (GSC)

4 GSC self renewal

4 NESTIN, SOX2, CD44

4 VEGFA

4 Vasculogenic mimicry

$\downarrow$ Proliferation

\section{Figure 5}

A schematic representation showing role of $\mathrm{NOTCH} 1$ and WNT5A coregulation in modulating stem cell characteristics and vasculogenic mimicry. GSCs belonging to mesenchymal subtype express high levels of NOTCH1 and WNT5A. Knockdown of either NOTCH1 or WNT5A results in suppressed stem cell markers such as NESTIN, SOX2 and CD44 and self-renewal property. Reduced levels of NOTCH1 and WNT5A drives GSCs towards proliferation and stem cell exhaustion along with negatively regulating vasculogenic mimicry potential. 


\section{Supplementary Files}

This is a list of supplementary files associated with this preprint. Click to download.

- SupplementaryInformation.pdf 